\title{
QUANTIZATION OF THE ELECTRIC CONDUCTIVITY IN CARBON NANOTUBES
}

\author{
M.A. GRADO-CAFFARO* and M. GRADO-CAFFARO \\ Sapienza S.L. Scientific Consultants, C/Julio Palacios 11, 9-B, 28029-Madrid, \\ Spain
}

(Received 16 April 2001; In final form 5 May 2001)

In this paper, the electric conductivity of carbon nanotubes is investigated by deriving a mathematical expression for the quantized conductance in an ideal one-dimensional potential well with a single electron moving in it. Our results are compared with experimental data.

Keywords: Quantized conductance; Carbon nanotubes; Potential well; Single electron

\section{INTRODUCTION}

Quantization of the electric conductance in an ideal one-dimensional potential well with a single electron constitutes a subject of great interest because this fact can be conceived as the origin of the quantization in other relatively complicated structures which are typical subjects of the physics of condensed matter. From the point of view of theoretical physics, the consideration of a simple one-dimensional quantum box for studying electric conductivity due to a charged single particle constitutes a fruitful starting point in order to develop further elaborated schemes suitable for concrete problems of applied solid-state physics; in this respect, we refer to nanostructures and other similar systems.

With respect to carbon nanotubes, certain theoretical studies predict that these tubes should exhibit ballistic conductance of electrons so that we may speak of a waveguide behaviour (see, for instance, refs. [1-5]).

*Corresponding author. 
On the other hand, the mean free path of the electrons is much longer than the length of a given nanotube and scattering should consist of elastic collisions so that some theoretical results [6-8] indicate that conductance would jump in multiples of the conductance quantum $G_{0}=2 e^{2} / h$ ( $e$ is the electron charge and $h$ is the Planck's constant). Nevertheless, these results disagree with experimental observations [9] which indicate that the first main conductance plateau takes place at $0.5 G_{0}$ or $1 G_{0}$ but never at $2 G_{0}$ as the second conductance value predicted in refs. [6-8]; the above experimental and theoretical studies refer to multiwalled carbon nanotubes except [6-8] in which only singlewalled nanotubes have been treated. At any rate, as it has been claimed in ref. [9], the authors [6-8] should infer that, for multiwalled nanotubes, conductance jumps in multiples of $G_{0}$. The aim of the present paper is to establish a mathematical relationship for the conductance of a one-dimensional conductor with a single electron; this formulation constitutes the basis to treat systems as the carbon nanotubes.

\section{THEORY}

Let us consider an ideal one-dimensional potential well of length $a$. It is well-known that the total energy of a single electron inside the well is quantized and it is given by $E_{n}=n^{2} \pi^{2} \hbar^{2} /\left(2 m a^{2}\right)$ where $m$ is the free-electron mass. Then, we can write:

$$
E_{n}=\frac{n^{2} \pi^{2} \hbar^{2}}{2 m a^{2}}=\frac{1}{2} m v_{n}^{2} \quad(n=1,2, \ldots)(\hbar=h /(2 \pi))
$$

since the potential energy is identically null along the conductor; $v_{n}$ denotes the magnitude of the electron velocity.

By virtue of Eq. (1), we may determine the transit time of the electron as follows:

$$
\tau_{n}=\int_{0}^{a} \frac{d x}{v_{n}}=\frac{a}{v_{n}}=\frac{2 m a^{2}}{n h}
$$

On the other hand, the conductivity in the well is given by:

$$
\sigma_{n}=\frac{e^{2} \tau_{n} \bar{N}_{n}}{m}
$$


where $\bar{N}_{n}$ denotes electron spatial density and $\tau_{n}$ is the relaxation time which here coincides exactly with the transit time; $\bar{N}_{n}$ is the average value of $N_{n}(x)$ where $N_{n}(x)=k \psi_{n}^{2}(x), k$ and $\psi_{n}(x)$ being a positive real constant and wavefunction, respectively $\left(\psi_{n}(x)=\left(\frac{2}{a}\right)^{1 / 2} \sin \left(\frac{n \pi x}{a}\right)\right)$. A being the cross-sectional area of the conductor, then we have $A \int_{0}^{a} N_{n}(x) d x=A k \int_{0}^{a} \psi_{n}^{2}(x) d x=A k \cdot 1=A k=1$ (one-electron system) so that $k=1 / A$ and we have:

$$
\bar{N}_{n}=\frac{1}{a} \int_{0}^{a} N_{n}(x) d x=\frac{1}{a A} \int_{0}^{a} \psi_{n}^{2}(x) d x=\frac{1}{a A}
$$

Notice that in formula (3) $\tau_{n}$ is, by definition, an average quantity so that the electron spatial density must be also considered as an average value in accordance with the fact that $\sigma_{n}$ is also conceived as an averaged quantity in our context. In addition, note the role played by the bound Schrödinger states $\psi_{n}(x)$ associated with the motion of the single electron in question.

Now, by combining formulae (2), (3) and (4), it follows:

$$
\sigma_{n}=\frac{2 a e^{2}}{A n h}
$$

so that the conductance is:

$$
G_{n}=\frac{\sigma_{n} A}{a}=\frac{2 e^{2}}{n h} \quad(n=1,2, \ldots)
$$

and, since $G_{0}=2 e^{2} / h$, from Eq. (6) it follows:

$$
G_{n}=G_{0} / n
$$

For $n=1$ (ground state) one has $G_{1}=1 G_{0}$ and for $n=2$ (first excited state) we have $G_{2}=0.5 G_{0}$; these values agree with the two values observed in ref. [9]. Formula (7) expresses that conductance is quantized and does not depend upon $a$ and $A$; at this point, we want to remark that, although the conductor is considered here as onedimensional, $A$ must be considered under the condition $A^{1 / 2} \ll a$ (a very thin conductor). 


\section{CONCLUSIONS}

We have performed a simple formulation for $G_{n}$ which, in spite of its simplicity, is adequate to simulate electric conduction in carbon nanotubes since our results agree with ref. [9]. The authors of ref. [9] used nanotubes with a width of $15 \mathrm{~nm}$ and a length of $4 \mu \mathrm{m}$; the conductance of multiwalled nanotubes seems to be scaled with respect to the number of involved layers [8,9]. In addition, we can mention that the tubes of ref. [9] typically have 5 to 30 layers. Finally, we want to remark that our approach can be regarded as a semiclassical formulation.

\section{References}

[1] T. W. Odom, J.-L. Huang, P. Kim and C. M. Lieber (1998). Atomic structure and electronic properties of single-walled carbon nanotubes. Nature 391, 62.

[2] U. Landman, W. D. Luedtke, N. A. Burnham and R. J. Colton (1990). Atomistic mechanisms and dynamics of adhesion, nanoindentation, and fracture. Science 248, 454.

[3] D. P. E. Smith (1995). Quantum point contact switches. Science 269, 371.

[4] S. Datta (1995). Electronic Transport Properties in Mesoscopic Systems (Cambridge University Press, Cambridge).

[5] H. Dai, E. W. Wong and C. M. Lieber (1996). Probing electrical transport in nanomaterials: conductivity of individual carbon nanotubes. Science 272, 523.

[6] L. Chico, L. X. Benedict, S. G. Louie and M. L. Cohen (1996). Quantum conductance of carbon nanotubes with defects. Phys. Rev. B 54, 2600.

[7] W. Tian and S. Datta (1994). A Haronov-Bohm-type effect in graphene tubules: a Landauer approach. Phys. Rev. B 49, 5097.

[8] M. F. Lin and K. W.-K. Shung (1995). Magnetoconductance of carbon nanotubes. Phys. Rev. B 51, 7592.

[9] S. Frank, P. Poncharal, Z. L. Wang and W. A. de Heer (1998). Carbon nanotube quantum resistors. Science 280, 1744-1746. 

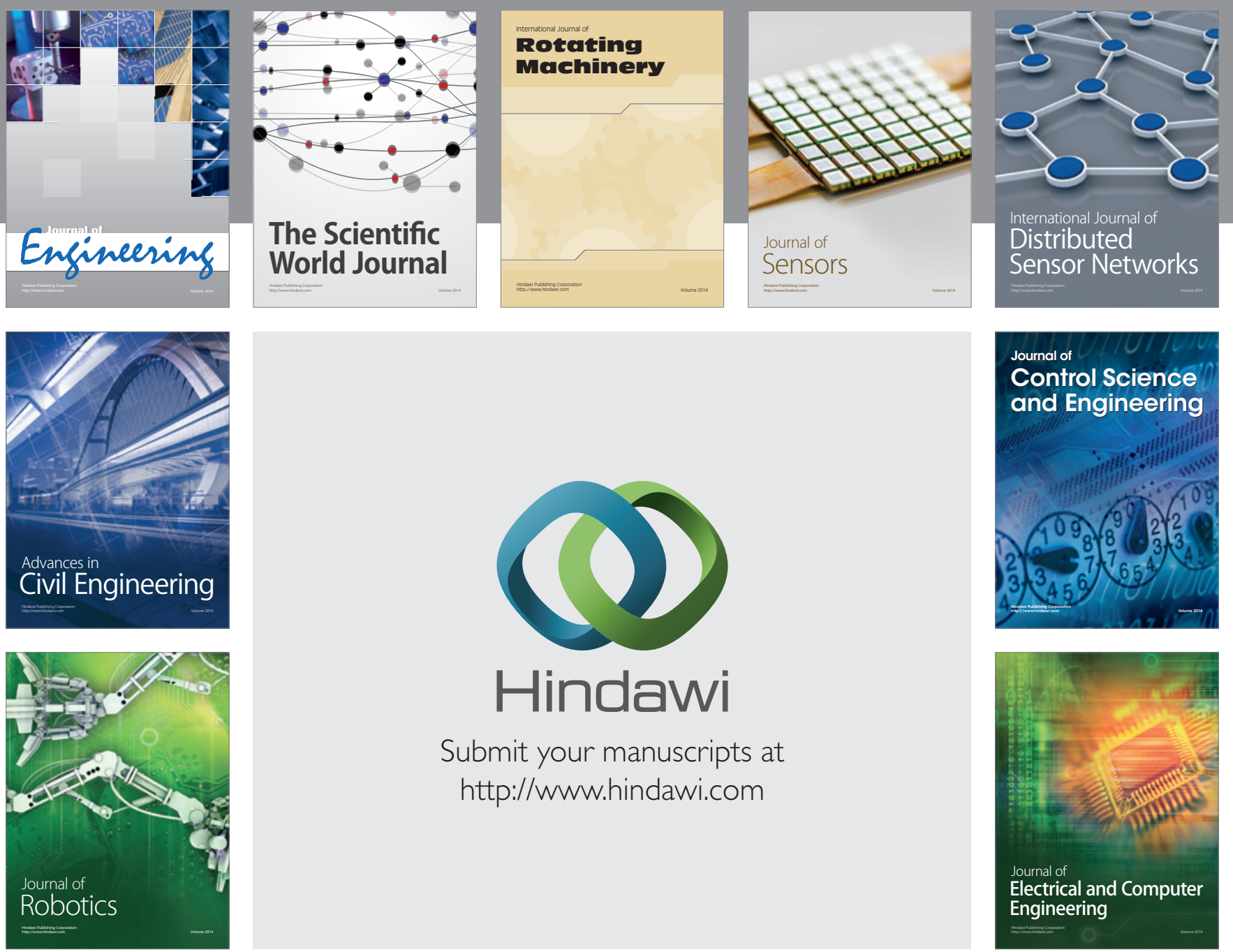

Submit your manuscripts at

http://www.hindawi.com
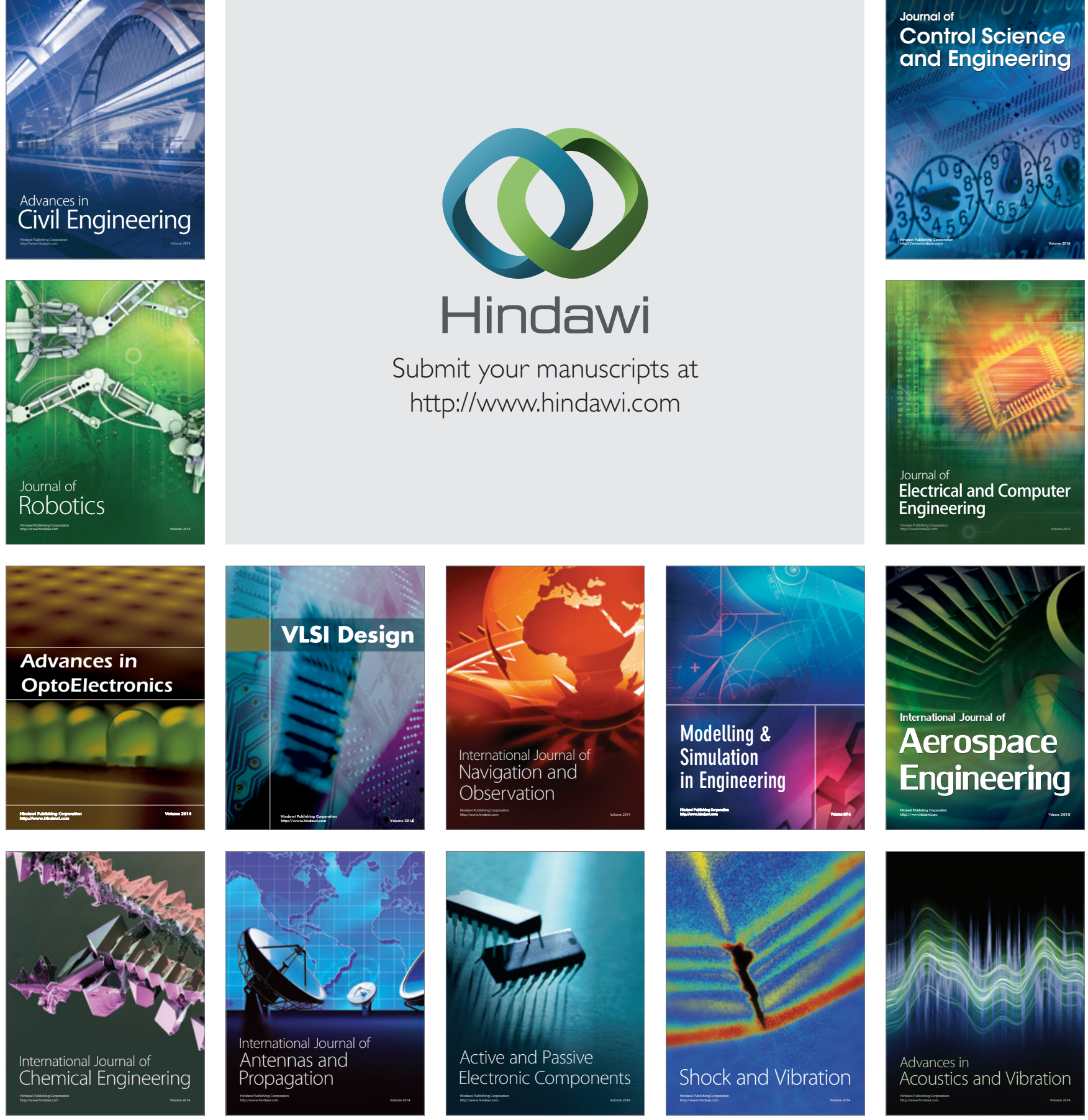